\title{
Knock-on effect of periodontitis to the pathogenesis of Alzheimer's disease?
}

\author{
Friedrich Leblhuber · Julia Huemer · Kostja Steiner · Johanna M. Gostner · Dietmar Fuchs
}

Received: 26 November 2019 / Accepted: 7 March 2020 / Published online: 25 March 2020

(C) The Author(s) 2020, corrected publication 2020

\begin{abstract}
Summary
Background Alzheimer's disease has chronic inflammatory components, which can be enhanced by systemic immune activation resulting in inflammation or vice versa. There is growing evidence that chronic periodontitis drives systemic inflammation and finally Alzheimer's disease. Thus, a link might exist between oral pathogens and Alzheimer's disease. This may be of special significance as there is an age-related incidence of chronic periodontitis.

Methods In this study, 20 consecutive patients with probable Alzheimer's disease were investigated. Diag-
\end{abstract}

\footnotetext{
The results of this study have been presented earlier at the $38^{\text {th }}$ International Winter-Workshop Clinical, Chemical and Biochemical Aspects of Pteridines and Related Topics in Innsbruck, February $26^{\text {th }}$-March 1st, 2019 and were published in abstract form (Pteridines [35]).
}

\section{F. Leblhuber $\cdot$ K. Steiner}

Department of Gerontology, Kepler University Clinic, Linz, Austria

\section{F. Leblhuber}

friedrich.leblhuber@liwest.at

\section{K. Steiner \\ kostja@kostja.com}

\section{J. Huemer}

Freelance Certified Dental Hygienist, Linz, Austria julia@huemer.at

\section{J. M. Gostner}

Division of Medical Biochemistry, Biocenter, Innsbruck

Medical University, Innsbruck, Austria

johanna.gostner@i-med.ac.at

\section{Fuchs $(\bowtie)$}

Division of Biological Chemistry, Biocenter, Innsbruck

Medical University, Innrain 80, 4th Floor, Room

M04-313, 6020 Innsbruck, Austria

dietmar.fuchs@i-med.ac.at nosis was established by cognitive tests, routine laboratory tests and cerebral magnetic resonance tomography. In $35 \%$ of these patients with cognitive impairment pathogenic periodontal bacteria were found.

Results The presence of Porphyromonas gingivalis, the key pathogen and one of the species involved in chronic periodontitis, was found to be associated with lower mini mental state examination scores $(p<0.05)$ and with a tendency to lower scores in the clock drawing test $(p=0.056)$. Furthermore, association between lower serum concentrations of the immune biomarker neopterin and the presence of Treponema denticola $(p<0.01)$ as well as of kynurenine were found in Alzheimer patients positive vs. negative for Tannerella forsytia $(p<0.05)$.

Conclusions Data indicate a possible association of specific periodontal pathogens with cognitive impairment, Treponema denticola and Tannerella forsytia may alter the host immune response in Alzheimer's disease. Albeit still preliminary, findings of the study may point to a possible role of an altered salivary microbiome as a causal link between chronic periodontitis and cognitive impairment in Alzheimer's disease.

Keywords Alzheimer's dementia - Cognitive decline · Neopterin · Neuroinflammation · Pathogenic oral bacteria

\section{Introduction}

In Alzheimer's disease (AD), the most common form of sporadic dementia with a complex multifactorial etiology including periodontal bacteria [1], neuroinflammation is an early event which can be exacerbated by systemic inflammation [2]. Increased neopterin production and tryptophan breakdown were found to be sensitive biomarkers of immune activation, 
Table 1 Clinical characteristics, psychometric and immunological data in 20 AD patients tested for periodontopathological bacteria

\begin{tabular}{|c|c|c|c|c|c|c|c|c|c|c|c|}
\hline $\begin{array}{l}\text { Patient } \\
\text { ID }\end{array}$ & Sex & $\begin{array}{l}\text { Age } \\
\text { (years) }\end{array}$ & $\begin{array}{l}\text { Trp } \\
\mu \mathrm{M}\end{array}$ & $\begin{array}{l}\text { Kyn } \\
\mu \mathrm{M}\end{array}$ & $\begin{array}{l}\mathrm{K} / \mathrm{T} \\
\mu \mathrm{M} / \mathrm{mM} \mathrm{nM}\end{array}$ & $\mathrm{NeO}$ & Trep-d & Tann-f & Por-g & MMSE & CDT \\
\hline KR & Male & 86 & 56.4 & 1.84 & 32.7 & 12.5 & 0 & 0 & 0 & 23 & 7 \\
\hline KR & Female & 63 & 71.0 & 2.08 & 29.3 & 9.6 & 0 & 0 & 0 & 29 & 7 \\
\hline $\mathrm{KP}$ & Male & 86 & 51.3 & 1.53 & 29.9 & 8.5 & 0 & 0 & 0 & 25 & 9 \\
\hline$A G$ & Male & 79 & 58.4 & 1.11 & 19.1 & 4.5 & 0 & 0 & 0 & 27 & 9 \\
\hline GR & Male & 76 & 56.0 & 1.91 & 34.2 & 7.5 & 0 & 0 & 0 & 25 & 5 \\
\hline IL & Male & 74 & 43.4 & 1.31 & 30.3 & 6.1 & 1 & 1 & 0 & 24 & 7 \\
\hline $\mathrm{FB}$ & Male & 79 & 57.2 & 2.53 & 44.2 & 7.8 & 1 & 1 & 1 & 3 & 3 \\
\hline$A Z$ & Female & 92 & 58.6 & 1.76 & 30.0 & 5.9 & 0 & 0 & 0 & 14 & 3 \\
\hline JS & Female & 78 & 51.8 & 2.03 & 39.1 & 9.3 & 0 & 0 & 0 & 17 & 7 \\
\hline WT & Male & 72 & 57.5 & 1.81 & 31.4 & 8.2 & 0 & 0 & 0 & 29 & 9 \\
\hline SF & Male & 87 & 70.4 & 3.58 & 50.9 & 14.0 & 0 & 0 & 0 & 21 & 7 \\
\hline $\mathrm{RN}$ & Female & 86 & 53.0 & 2.09 & 39.4 & 8.5 & 0 & 0 & 0 & 21 & 7 \\
\hline $\mathrm{JH}$ & Male & 73 & 73.0 & 2.86 & 39.2 & 13.6 & 0 & 0 & 0 & 27 & 9 \\
\hline WP & Male & 72 & 48.3 & 1.29 & 26.6 & 4.9 & 1 & 1 & 1 & 18 & 3 \\
\hline KR & Male & 76 & 62.8 & 1.76 & 28.0 & 7.4 & 1 & 1 & 1 & 8 & 0 \\
\hline MM & Female & 73 & 69.7 & 1.77 & 25.4 & 8.9 & 0 & 1 & 0 & 25 & 7 \\
\hline $\mathrm{MB}$ & Female & 56 & 54.7 & 1.18 & 21.6 & 4.5 & 1 & 1 & 1 & 24 & 9 \\
\hline LH & Female & 97 & 66.7 & 2.14 & 32.1 & 8.3 & 0 & 0 & 0 & 14 & 0 \\
\hline $\mathrm{HP}$ & Female & 69 & 36.6 & 1.67 & 45.7 & 13.3 & 0 & 1 & 1 & 14 & 0 \\
\hline TH & Female & 87 & 53.1 & 3.38 & 63.6 & 11.1 & 0 & 0 & 0 & 21 & 7 \\
\hline Mean & - & 78.1 & 57.5 & 1.98 & 34.6 & 8.7 & - & - & - & 20.5 & 5.8 \\
\hline
\end{tabular}

which are derived from stimulation of indoleamine 2,3-dioxygenase-1 (IDO) and GTP(guanosine triphosphate)-cyclohydrolase 1 by interferon-gamma [3, 4]. Similar changes albeit to a lower degree can be observed in a large percentage of older individuals due to the age-related immune response even if healthy [5], but more often and to a greater extent in $A D$ [6-8]. Especially the tryptophan metabolism could play a major role because the decrease of tryptophan concentrations and the parallel increase of the neurotoxic tryptophan catabolite quinolinic acid were observed to relate to impaired cognitive abilities of patients [2, 8].

A high percentage of older people suffer from periodontitis, the prevalence of which increases with age [9]. Interestingly, periodontal disease was found to be associated with higher brain amyloid load even in healthy older persons [10]. In recent studies, a close and causal link between chronic periodontitis, aggravating systemic inflammation and cognitive impairment was hypothesized [11, 12]. This hypothesis is underlined by recent preclinical data [13].

Porphyromonas gingivalis is described as the key pathogen which causes polymicrobial synergy and dysbiosis gaining greater virulence [14]. Different periodontopathogenic bacterial strains are described to correlate with inflammatory mediators and $\mathrm{AD}$ [15].
Dysbiosis of intestinal microbiota in old people has earlier been described to cause leaky gut, which results in silent systemic inflammation and via the microbiota-gut-brain axis in neuroinflammation, a relevant pathomechanism in the course of AD [2, 16-19]. Poor dental status and periodontal disease has earlier been linked to reduced cognitive function and $A D$ [20]. In the Nun study, participants with the fewest

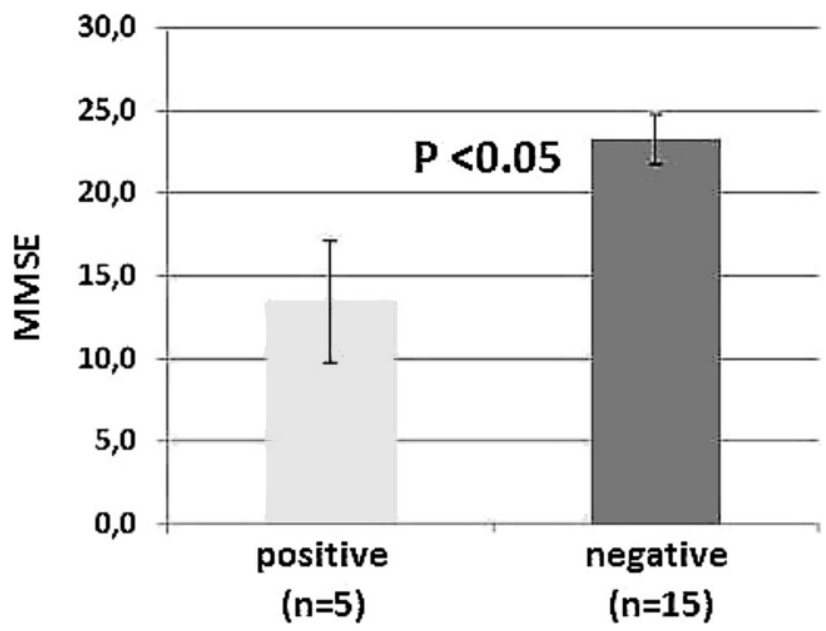

Fig. 1 Patients with $A D$ positive for Porphyromonas gingivalis present with statistically lower scores in the mini mental state examination test (MMSE; left) 

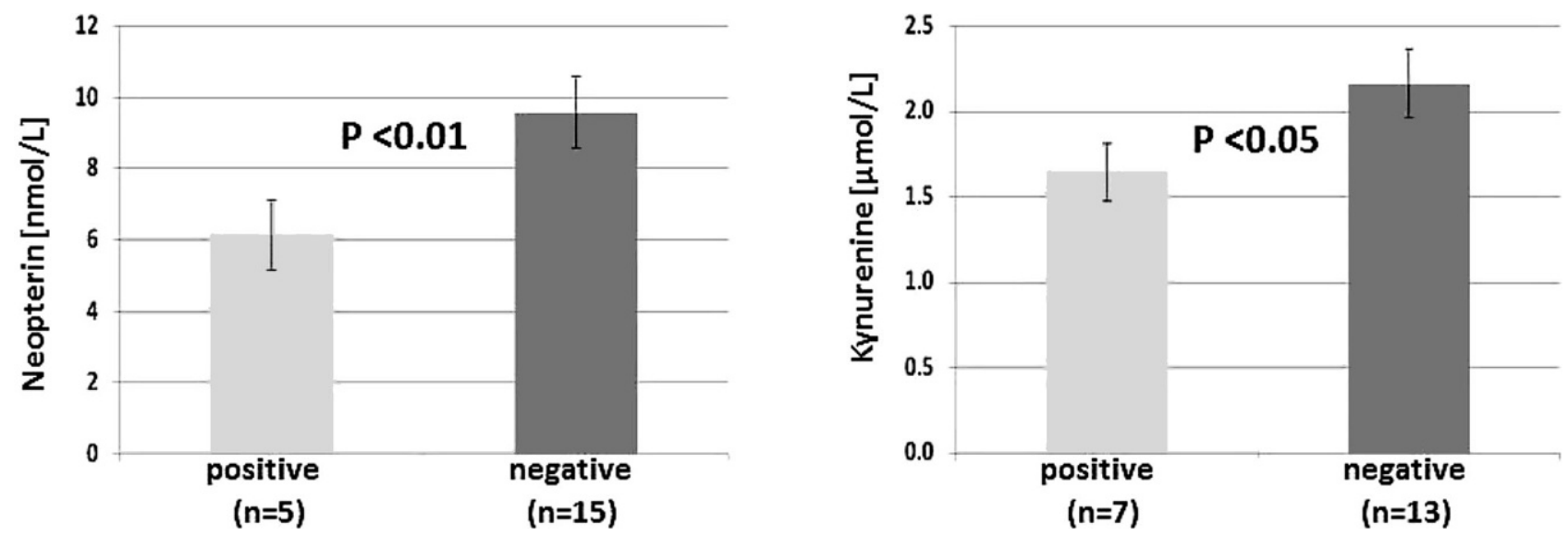

Fig. 2 Lower mean values \pm SEM of serum neopterin (left) and kynurenine (right) concentrations in $A D$ patients positive for Treponema denticola (left) and Tannerella forsythia (right) respectively

teeth had the highest risk of prevalence and incidence of dementia [21, 22]. In an earlier postmortem study, pathogenic periodontal disease bacteria, Treponema denticola, Tannerella forsythia, and Porphyromonas gingivalis, were identified in brain tissue indicating a link between chronic periodontal disease and $\mathrm{AD}$ [23].

\section{Patients, material and methods}

After written informed consent, the diagnosis of primary degenerative dementia (F 00.1, in accordance with the International Classification of Diseases-ICD10) was established in 20 patients by cerebral magnetic resonance imaging (MRI) and routine laboratory tests as described earlier $[19,24]$. Cognitive testing was performed by mini mental state examination (MMSE) and clock drawing test (CDT).

The following laboratory parameters were also analyzed: serum concentrations of neopterin and of tryptophan and kynurenine, calculating the kynurenine to tryptophan ratio (Kyn/Trp) as an index of tryptophan breakdown [4, 24]. Additionally, alveolar fluid was tested by RNA-based analysis (PerioPOC ${ }^{\circledR}$, Genspeed Biotech, Henry Schein Dental, Vienna, Austria) for the presence of the following periodontal pathogens: Treponema denticola, Tannerella forsythia, Porphyromonas gingivalis, Prevotella intermedia and Aggregatibacter actinomycetemcomitans [25].

Data were analyzed by the Statistical Package for Social Science (version 22, IBM Statistics SPSS, Chicago, IL, USA). To take into account that not all collected data followed a normal distribution, nonparametric Friedman and Wilcoxon signed-rank test were applied. To test for associations between variables, Spearman rank correlation analysis was performed, and $p$ values below 0.05 were considered to indicate statistical significance.

\section{Results}

From 55 outpatients of the Department of Gerontology of the Neuromed Campus at the Kepler University Clinic Upper Austria with different neuropsychiatric symptomatology, 20 consecutive patients (aged $78.1 \pm 2.2$ years, 9 females) with symptoms of cognitive impairment were recruited. None of them were smokers.

The procedure was well-tolerated by all patients. In MRI scans all patients showed global cerebral atrophy without circumscribed vascular lesions. The mean \pm SD MMSE in the patients was: $20.5 \pm 7.0$ and the CDT scores were $5.8 \pm 3.1$.

In seven of the patients investigated with clinical signs of periodontitis stage III and IV [26], pathological periodontitis strains were found: T. forsythia in one, T. denticola and T. forsythia together in one, T. denticola, T. forsythia and P. gingivalis together in five patients; these also showed the lowest MMSE and CDT scores, see Table 1. P. intermedia and A. actinomycetemcomitans could be detected in none of the cases studied.

As a main result in this investigative study, a significant association was observed between the salivary presence of $P$. gingivalis and lower MMSE (positive: $13.4 \pm 3.68$; vs. negative: $23.3 \pm 1.50$; $U=2.239$, $p<0.05$; Fig. 1). There was also a tendency to lower scores in the CDT (positive: $3.00 \pm 1.64$; vs. negative: $7.1 \pm 0.73$; $U=1.989, p=0.056$; Table 1 ) when this particular pathogen was present.

Furthermore, the presence of $T$. denticola was associated with lower serum neopterin concentrations (positive: $6.14 \pm 0.65$ vs. negative: $9.58 \pm 0.73 \mathrm{nmol} / \mathrm{L}$; $\mathrm{U}=2.533, p<0.01$ ); the presence of $T$. forsythia resulted in lower serum kynurenine concentrations compared to a negative saliva test result (positive $1.64 \pm 0.17$ vs. negative: $2.16 \pm 0.20 ; U=1.980, p<0.05$; Fig. 2 ; Table 1).

In the whole data set, a significant positive correlation existed between serum concentrations of 
neopterin and Kyn/Trp (rs $=0.674, p<0.001$ ) confirming earlier results $[2,27]$.

\section{Discussion}

Results of the present series confirm that chronic low-grade immune activation and inflammation alter monoamine metabolism that may be involved in the development of neuropsychiatric symptoms characteristic for age and dementia $[2,5,27,28]$. The present exploratory pilot study described the presence of the periodontal pathogenic bacteria $T$. denticola, T. forsythia, and $P$. gingivalis in saliva of a subgroup of demented patients causing chronic oral inflammation as mentioned earlier $[19,29]$. P gingivalis, the most virulent pathogen of the bacteria associated with periodontitis [14] with remote body inflammatory pathologies, was found to be associated with lower MMSE $(p<0.05$; Fig. 1$)$ and also albeit only weakly with lower CDT scores $(p<0.06)$ in the patients indicating a possible link between this periodontal pathogenic strain and neuroinflammation and the dementia process.

In an animal study published recently [13], experimental chronic periodontitis was induced by repeated oral application of $P$. gingivalis. Gingipain, a protease secreted by this bacterium, could be detected immunohistochemically in the hippocampi of experimental mice confirming its translocation to the brain. Also, signs of neuroinflammation, neurodegeneration and the formation of extracellular $\mathrm{A}_{42}$ (myloid -peptide 42) consistent with neuropathological findings in $\mathrm{AD}$ could be shown after repeated oral application of $P$. gingivalis in young adult wild type mice [13]. In AD, P. gingivalis possibly affects the blood-brain barrier permeability and influences local IFN- $\gamma$ response by preventing entry of immune cells into the brain. The scarcity of adaptive immune cells in $\mathrm{AD}$ neuropathology implies $P$. gingivalis infection of the brain likely causing impaired clearance of insoluble amyloid and inducing immunosuppression [14, 30]. An inverse association was seen between the presence of salivary $T$. denticola and concentrations of neopterin $(p<0.01)$ as well as between salivary $T$. forsythia and kynurenine $(p<0.05)$. These results probably indicate that these pathogens may counteract adaptive (Th1 type [ $T$ helper cells type 1]) immunity by triggering regulatory $\mathrm{T}$-cells which finally may suppress and downregulate the inflammation process as recently hypothesized $[2,20]$. Likewise, $P$. gingivalis was reported to suppress adaptive immunity in $\mathrm{AD}$ patients [30]. Probably an imbalance of immune functions and an imbalance of adaptive immunosuppression could be caused by these pathogenic bacterial species; however, associations between the presence or absence of periodontal pathogens and abnormal concentrations of immune system biomarkers neopterin and/or kynurenine were only seen for 3 pathogens, namely T. denticola, T. forsythia and P. gingivalis, but not for
P. intermedia and A. actinomycetemcomitans. This result, however, can only be regarded as preliminary because of the small number of patients investigated thus far.

Interestingly, six of the patients positive for periodontal pathogens were ApoE4 (apolipoprotein E4) allele carriers, two of them homozygous; in an earlier study, individuals with both a low number of teeth and the ApoE4 allele performed worse in cognitive tests an cd showed more rapid cognitive decline with time [31]. The data set in this study was too small for detailed statistical analysis, and future studies will be necessary to address this issue properly.

To conclude, in the absence of longitudinal data the preliminary findings can only provide correlational evidence that periodontal bacterial infections may be additive in the pathogenesis of cognitive impairment and AD. Any correlation-even if significant-cannot be interpreted as cause-effect relationship in any direction. It only indicates an association between the events. Future longitudinal studies including periodontal disease in larger numbers of dementia patients as well as age-related non-dementia individuals [10] with respect to the ApoE status are necessary to elucidate the specific role of the oral microbiome in neuroinflammation and neurodegeneration and its potential to prevent or delay the onset of $\mathrm{AD}$. The presence of specific pathogens relating to immunobiochemical changes in patients could probably reflect the fact that these patients are no longer able to perform sufficient dental hygiene. Additional longitudinal studies also should investigate-beyond sufficient dental hygiene [14] - the effects of the consumption of a certain bacterial strain to improve or prevent periodontal disease in the aging population [32]. Small molecule inhibitors to reduce the bacterial load of certain bacteria like $P$. gingivalis will be developed in the near future [33]. Thus, encompassing investigation of the symbiotic intestinal, oral as well as nasal microbiome might give additional information to define new pathways for evolutionary AD treatment [34].

This study is certainly limited by the relatively small number of patients. Thus, results can only be regarded as preliminary; however, they provide some new aspect that the salivary microbiome could play a relevant role in the pathophysiology of $\mathrm{AD}$. It might also draw attention to a potential bottom-up contribution in $\mathrm{AD}$ which is usually regarded to be top-down only. With this respect it appears attractive that saliva can be easily assessed with no burden to the patients but enables monitoring of a potentially important aspect in the pathophysiology of $\mathrm{AD}$.

Funding Open access funding provided by University of Innsbruck and Medical University of Innsbruck. 


\section{Compliance with ethical guidelines}

Conflict of interest F. Leblhuber, J. Huemer, K. Steiner, J.M. Gostner, and D. Fuchs declare that they have no competing interests.

Ethical standards The study was performed in accordance with the criteria of the Declaration of Helsinki and approved by the local ethics committee < dated $2017 / 05 / 11$ by the Ethikkommittee des Landes Oberösterreich, Studie Nr. I-24$16>$. Human specimens were collected following an approved study protocol. The work does not report experiments involving animals.

Open Access This article is licensed under a Creative Commons Attribution 4.0 International License, which permits use, sharing, adaptation, distribution and reproduction in any medium or format, as long as you give appropriate credit to the original author(s) and the source, provide a link to the Creative Commons licence, and indicate if changes were made. The images or other third party material in this article are included in the article's Creative Commons licence, unless indicated otherwise in a credit line to the material. If material is not included in the article's Creative Commons licence and your intended use is not permitted by statutory regulation or exceeds the permitted use, you will need to obtain permission directly from the copyright holder. To view a copy of this licence, visit http://creativecommons.org/licenses/by/4.0/.

\section{References}

1. Pritchard AB, Crean S, Olsen I, Singhrao SK. Periodontitis, microbes and their role in Alzheimer's disease. Front Aging Neurosci. 2017;24(9):336.

2. Leblhuber F, Strasser B, Steiner K, Gostner J, Schuetz B, Fuchs D. On the role of intestinal microbiota in patients with cognitive decline. JPharm Pharmacol. 2017;5:648-53.

3. Murr C, Widner B, Wirleitner B, Fuchs D. Neopterin as a marker for immune system activation. Curr Drug Metab. 2002;3:175-87.

4. Schroecksnadel K, Wirleitner B, Winkler C, Fuchs D. Monitoring tryptophan metabolism in chronic immune activation. Clin Chim Acta. 2006;364:82-90.

5. Capuron L, Schroecksnadel S, Féart C, Aubert A, HigueretD, Barberger-Gateau P, et al. Chronic low grade immune activation in the elderly is associated with increased tryptophan catabolism and altered phenylalanine turnover: role in neuropsychiatric symptomatology. Biol Psychiatry. 2011;70:175-82.

6. Sawada M, Hirata Y, Arai H, Iizuka R, Nagatsu T. Tyrosine hydroxylase, tryptophan hydroxylase, biopterin, and neopterin in the brains of normal controls and patients with senile dementia of Alzheimer type. J Neurochem. 1987;48(3):760-4.

7. Hamon CG, Cattell RJ, Wright CE, Wychrij O, Blair JA, Harding GF. Visual evoked potentials and neopterin: biopterin ratios in urine show a high correlation in Alzheimer's disease. J Neurol Neurosurg Psychiatry. 1988;51(2):314-5.

8. Giil LM, Midttun Ø, Refsum H, UlvikA, Advani R, Smith AD, et al. Kynurenine pathway metabolites in Alzheimer's disease. JAlzheimers Dis. 2017;60(2):495-504.

9. $\mathrm{Wu} \mathrm{Z}$, Nakanishi H. Connection between periodontitis and Alzheimer's disease: possible roles of microglia and leptomeningeal cells. J PharmacolSci. 2014;126:8-13.

10. Kamer AR, Pirraglia E, Tsui W, Rusinek H, Valiabhajosula S, Mosconi L, et al. Periodontal disease associates with higher brain amyloid load in normal elderly. Neurobiol Aging. 2015;36(2):627-33.

11. Shoemark DK, Allen SJ. The microbiome and disease: reviewing the links between the oral microbiome, aging, and Alzheimer's disease. J Alzheimers Dis. 2015;43(3):725-34.

12. Sochocka M, Sobczynski M, Sender-Janeczek A, Zwolińska K, Błachowicz O, Tomczyk T, et al. Association between periodontal health status and cognitive abilities. The role of cytokine profile and systemic inflammation. Curr Alzheimer Res. 2017;14(9):978-90.

13. Ilievsky V, Zuchowska PK, Green SJ, Toth PT, Ragozzino ME, Le $\mathrm{K}$, et al. Chronic oral application of a periodontal pathogen results in brain inflammation, neurodegeneration and amyloid beta production in wild type mice. PLoS ONE. 2018;13(10):e204941.

14. Harding A, Robinson S, Crean S, Singhrao SK. Can better management of periodontal disease delay the onset and progression of Alzheimer's disease? J Alzheimers Dis. 2017;58:337-48.

15. Noble JM, Scarmeas N, Celenti RS, Elkind MS, Wright CB, SchupfN, etal. SerumIgGantibodylevels to periodontalmicrobiota are associated with incident Alzheimer's disease. Plos One. 2014;9:e114959.

16. Caracciolo B, Xu W, Collins S, Fratiglioni L. Cognitive decline, dietary factors and gut-brain interactions. Mech Ageing Dev. 2014;136-137:59-69.

17. MaheshwariP,EslickGD. BacterialinfectionandAlzheimer's disease. JAlzheimers Dis. 2015;43(3):957-66.

18. Minter MR, Zhang C, Leone V, Ringus DL, Zhang X, OylerCastrillo P, et al. Antibiotic-induced perturbations in gut microbial diversity influences neuroinflammation and amyloidosis in a murine model of Alzheimer's disease. Sci Rep. 2016;6:30028.

19. Leblhuber F, Steiner K, Schuetz B, Fuchs D, Gostner JM. Probiotic supplementation in patients with Alzheimer's dementia - an explorative intervention study. Curr Alzheimer Res. 2018;15(12):1106-13.

20. Tremlett H, Bauer KC, Appel-Cresswell S, Finlay BB, Waubant E. The gut microbiome in human neurological disease: a review. Ann Neurol. 2017;81:369-82.

21. Gatz M, Mortimer JA, Fratiglioni L, Johansson B, Berg S, Reynolds CA, et al. Potentially modifiable risk factors for dementia in identical twins. Alzheimers Dement. 2006;2:110-7.

22. Stein PS, Desrosiers M, Donegan SJ, Yepes JF, Kryscio RJ. Tooth loss, dementia and neuropathology in the nun study. JAmDent Assoc. 2007;138:1314-22.

23. Poole S, Singhrao SK, Kesavalu L, Curtis MA, Crean S. Determining the presence of periodontopathic virulence factors in short term postmortem Alzheimer's disease brain tissue. J Alzheimers Dis. 2013;36(4):665-77.

24. Widner B, Werner ER, Schennach H, Wachter H, Fuchs D. Simultaneous measurements of serum tryptophan and kynurenine by HPLC. Clin Chem. 1997;43:2424-6.

25. Polonyi M, Prenninger N, Arweiler NB, Haririan H, Winklehner P, Kierstein S. Assessment of viable periodontal pathogens by reverse transcription quantitative polymerase chain reaction. J Periodontal Res. 2013;48(5): 671-6.

26. Tonetti MS, Greenwell H, Kornman KS. Staging and grading of periodontitis: framework and proposal of a new classification and case definition. J Clin Periodontol. 2018;45(20):S149-S61.

27. Widner B, Leblhuber F, Walli J, Tilz GP, Demel U, Fuchs D. Tryptophan degradation and immune activation in Alzheimer's disease. J Neural Transm (Vienna). 2000;107:343-53. 
28. Neurauter G, Schröcksnadel K, Scholl-Bürgi S, SpernerUnterweger B, Schubert C, Ledochowski M, et al. Chronic immune stimulation correlates with reduced phenylalanine turnover. Curr Drug Metab. 2008;9(7):622-7.

29. Singhrao SK, Harding A, Simmons T, Robinson S, Kesavalu L, Crean S. Oral inflammation, toothloss, riskfactors, and association with progression of Alzheimer's disease. JAlzheimers Dis. 2014;42(3):723-37.

30. Olsen I, Taubman MA, Singhrao SK. Porhyromonas gingivalis suppresses adaptive immunity in periodontitis, atherosclerosis, and Alzheimer's disease. J Oral Microbiol. 2016;8:10.

31. Sparks Stein P, Steffen MJ, Smith C, Jicha G, Ebersole JI, Abner E, et al. Serum antibodies to periodontal pathogens areariskfactor for Alzheimer's disease. Alzheimers Dement. 2012;8(3):196-203.

32. Tobita K, Watanabe I, Tomokiyo M, Saito M. Effects of heat-treated Lactobacillus crispatus KT-11 strain consumption on improvement of oral cavity environment: a randomised double-blind clinical trial. Benef Microbes. 2018;9(4):585-92.

33. Dominy SS, Lynch C, Emini F, Benedyk M, Marczyk A, Konradi A, et al. Porphyromonas gyngivalis in Alzheimer's disease brains: evidence for disease causation and treatment with small molecule inhibitors. Sci Adv. 2019; https:// doi.org/10.1126/sciadv.aau3333.

34. Fox M, Knorr DA, Haptonstall KM. Alzheimer's disease and symbiotic microbiota: an evolutionary medicine perspective. Ann NYAcad Sci. 2019;1449(1):3-14.

35. Leblhuber F, Huemer J, Steiner K, Gostner JM, Fuchs D. Knock on effect from periodontitis to the pathogenesis of Alzheimer's disease? Pteridines 2019:30:90. Abstract of a presentation at the 38th International Winter-Workshop Clinical, Chemical and Biochemical Aspects of Pteridines and Related Topics, Innsbruck2019

Publisher's Note Springer Nature remains neutral with regard to jurisdictional claims in published maps and institutional affiliations. 